\title{
Inertia Compensation Control of a One-Degree-of- Freedom Exoskeleton for Lower-Limb Assistance: Initial Experiments
}

\author{
Gabriel Aguirre-Ollinger, Member, IEEE, J. Edward Colgate, Senior Member, IEEE, \\ Michael A. Peshkin, Senior Member, IEEE, and Ambarish Goswami, Senior Member, IEEE
}

\begin{abstract}
A new method of lower-limb exoskeleton control aimed at improving the agility of leg-swing motion is presented. In the absence of control, an exoskeleton's mechanism usually hinders agility by adding mechanical impedance to the legs. The uncompensated inertia of the exoskeleton will reduce the natural frequency of leg swing, probably leading to lower step frequency during walking as well as increased metabolic energy consumption. The proposed controller emulates inertia compensation by adding a feedback loop consisting of low-pass filtered angular acceleration multiplied by a negative gain. This gain simulates negative inertia in the low-frequency range. The resulting controller combines two assistive effects: increasing the natural frequency of the lower limbs and performing net work per swing cycle. The controller was tested on a statically mounted exoskeleton that assists knee flexion and extension. Subjects performed movement sequences, first unassisted and then using the exoskeleton, in the context of a computer-based task resembling a race. In the exoskeleton's baseline state, the frequency of leg swing and the mean angular velocity were consistently reduced. The addition of inertia compensation enabled subjects to recover their normal frequency and increase their selected angular velocity. The work performed by the exoskeleton was evidenced by catch trials in the protocol.
\end{abstract}

Index Terms-Admittance control, exoskeleton, rehabilitation robotics.

\section{INTRODUCTION}

A $\mathrm{N}$ EXOSKELETON is a wearable mechanism, usually with an anthropomorphic configuration, capable of tracking the movements of the user's extremities. In most applications, exoskeletons are designed to produce forces that assist the user in performing a motor task. Different types of lower-limb exoskeletons and powered orthoses are currently being developed as tools for gait rehabilitation and mobility assistance. Gait trainers are exoskeletons designed for physical

Manuscript received March 20, 2011; revised October 03, 2011; accepted November 12, 2011. Date of current version January 25, 2012. This research was supported in part by a grant from the Honda Research Institute, Mountain View, CA.

G. Aguirre-Ollinger is with the School of Electrical, Mechanical and Mechatronic Systems, University of Technology, Sydney, Broadway, NSW 2007, Australia (e-mail: gabriel.aguirre-ollinger@uts.edu.au).

J. E. Colgate and M. A. Peshkin are with the Department of Mechanical Engineering, Northwestern University, Evanston, IL 60208 USA.

A. Goswami is with the Honda Research Institute, Mountain View, CA 94041 USA.

Color versions of one or more of the figures in this paper are available online at http://ieeexplore.iee.org.

Digital Object Identifier 10.1109/TNSRE.2011.2176960 therapy and neurorehabilitation [1]-[3]. Another category is formed by autonomous wearable exoskeletons for human force augmentation. A common application for such devices is increasing the load-carrying capabilities of the user [4], [5]. Although these machines can reduce fatigue from carrying the load, in general they do not provide supplementary propulsion during walking.

Ferris [6] has postulated that a general goal in the design of lower-limb exoskeletons should be reducing the metabolic cost of walking. Independently of other functional goals, such as correction of the gait pattern, an exoskeleton should at least avoid increasing the metabolic cost with respect to unassisted walking. However, recent surveys show that very few of the existing exoskeleton systems have demonstrated any capability to reduce metabolic consumption [6]-[8]. In some cases, the device has actually produced the opposite effect [9]. A notable exception is the pneumatically-powered ankle-foot orthosis (AFO) developed by Ferris [10], which has achieved an economy of about $10 \%$ of the cost of walking unaided.

Another goal that has yet to be accomplished in exoskeleton research is improving the agility of the user's leg movements. For the case of level walking, agility can be defined as the maximum forward speed that can be sustained comfortably by the subject. Few studies have been conducted so far linking the use of an exoskeleton to the user's selected speed of walking [11]. Increasing agility by means of an exoskeleton poses a considerable challenge because the exoskeleton's impedance (i.e., the combined effects of the mechanism's mass, friction and weight) will tend to increase the metabolic cost of walking and to slow the user down [12], [13]. Actuation and control in the exoskeleton thus have to serve a dual purpose: making the exoskeleton's mechanism as transparent to the user as possible, and providing assistive forces to achieve the functional goal of the exoskeleton.

The authors have previously introduced the concept of making the assistive function of the exoskeleton a natural extension of the "transparency" controller's capabilities [14]. The concept has been tested on a stationary one-degree-of-freedom (1-DOF) exoskeleton designed to assist knee flexion and extension [15]. In the exoskeleton's baseline state, an admittance controller masks part of the dynamics of the mechanism from the user, namely the damping and the weight of the device. The same controller can turn the exoskeleton into an assistive device by making its virtual admittance active. In an earlier study, the authors tested the use of negative damping in order to make the exoskeleton perform net work on the legs [14], [16]. 
The present study is concerned with modifying the controller for the 1-DOF exoskeleton to emulate inertia compensation in the low-frequency range. The requisite of maintaining stability makes inertia compensation difficult to achieve. When admittance control is used, the presence of compliance in the mechanism impedes making the virtual inertia arbitrarily low [17]. Our proposed controller is conceived to counteract the inertia of the exoskeleton's mechanism and, by extension, perform a partial compensation of the inertia of the human limb. In our approach, inertia compensation is emulated through positive feedback of the exoskeleton's angular acceleration, low-pass filtered at a relatively low cutoff frequency. The use of positive feedback implies that the exoskeleton is unstable in isolation. However, a previous stability analysis [18] has shown that the coupled system formed by the human limb and the exoskeleton can in theory be stabilized by virtue of the passive dynamics of the human limb. The same analysis yielded a range of feedback gains that can produce a virtual reduction of the inertia of the human limb without loss of stability.

The results presented here represent the continuation of a previously reported study [18]. Robust stability conditions of the coupled system formed by the human limb and the exoskeleton are derived, and the influence of the human limb impedance parameters is analyzed. In the experimental part of the study, subjects performed multiple series of leg-swing movements in the context of a computer-based pursuit task. The experimental conditions included moving the leg unaided, and then doing so with the aid of the exoskeleton. The subjects' mean speed of leg swing and its components, swing frequency and swing amplitude, were initially affected by the inertia of the exoskeleton. The inertia-compensation controller was subsequently employed to counteract these inertial effects. The experiment included catch trials to verify that the speed of swing motion was due in part to net work performed by the exoskeleton. The present study focused on the "steady-state" behavior of leg swing with the exoskeleton. The question of how the exoskeleton affects the ability to initiate or stop movements (specifically the ability to impart acceleration on the legs) has been addressed in a separate study [19].

\section{METHODS}

\section{A. Design and Control of a 1-DOF Exoskeleton With Emulated Inertia Compensation}

The authors have designed and built a stationary 1-DOF exoskeleton for assisting knee flexion and extension exercises. Its purpose is to investigate the effects of the exoskeleton's virtual dynamics (i.e., the dynamics resulting from closed-loop control) on the kinematics of leg-swing motion. Fig. 1(a) shows the exoskeleton's main assembly, consisting of a servo motor, a cable-drive transmission and a pivoting arm. The cable-drive solution avoids the occurrence of backlash in the transmission, thereby eliminating the risk of limit cycles. The servo motor (AC servo from Kollmorgen, Radford, VA) and cable drive combination is capable of delivering a continuous torque output of about $20.0 \mathrm{~N}-\mathrm{m}$.

For actual use the exoskeleton assembly is mounted on a rigid support frame [Fig. 1(b)], and the subject uses the device in a

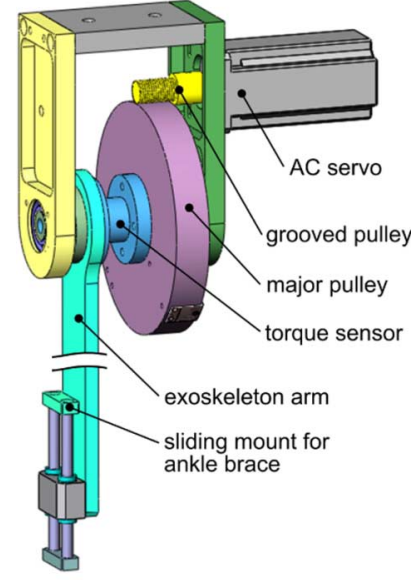

(a)

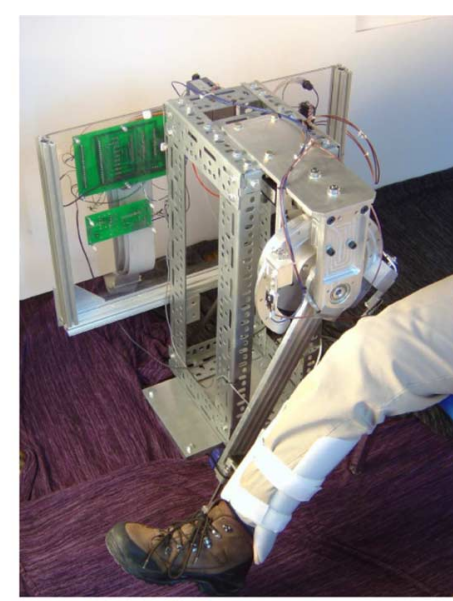

(b)
Fig. 1. Design of the stationary 1-DOF exoskeleton for knee flexion and extension. (a) Diagram of the exoskeleton's motor, cable drive and arm assembly. (b) 1-DOF exoskeleton in use.

seated position. A custom-built ankle brace couples the user's leg to the exoskeleton arm. The ankle brace is mounted on a sliding bracket in order to accommodate any possible radial displacement of the ankle relative to the device's center of rotation. Kinematic feedback consists of angular position and angular acceleration. The servo motor features emulated encoder output with a resolution of 131072 counts after quadrature. The angular acceleration of the exoskeleton arm is measured by means of an MT9 inertial measurement unit (IMU) from Xsens Technologies (Enschede, The Netherlands), operating at a sampling rate of $200 \mathrm{~Hz}$. A more detailed description of the exoskeleton's design is provided in a previous report [18].

\section{B. Assistance Through Admittance Control and Emulated Inertia Compensation}

Admittance control is employed to make the exoskeleton drive follow a virtual admittance model consisting of inertia moment $\bar{I}_{e}^{d}$, damping coefficient $\bar{b}_{e}^{d}$, and stiffness coefficient $\bar{k}_{e}^{d}$

$$
\bar{Y}_{e}^{d}(s)=\frac{s}{\bar{I}_{e}^{d} s^{2}+\bar{b}_{e}^{d} s+\bar{k}_{e}^{d}} .
$$

The most basic use of the admittance controller is to mask the dynamics of the exoskeleton arm from the user. The damping felt by the user can be canceled by making $\bar{b}_{e}^{d}=-b_{\text {arm }}$. The term $\bar{k}_{e}^{d}$ can be used to approximately balance the weight of the exoskeleton's arm. However, it is a simple matter to add a nonlinear term to the controller in order to produce a precise cancellation of the gravitational effects on the arm.

We have previously examined the question of whether admittance control can be used to compensate the inertia of the exoskeleton arm, or even the inertia of the human limb [18]. A stability analysis showed that it is not feasible to implement a negative inertia on the admittance controller and use the inertia of the human limb to guarantee stability. Noncollocation of the exoskeleton's actuator and the torque sensor will cause the coupled system to become unstable even for positive values of virtual inertia, if these are too low in magnitude. The proposed alternative is an approximate form of inertia compensation that 


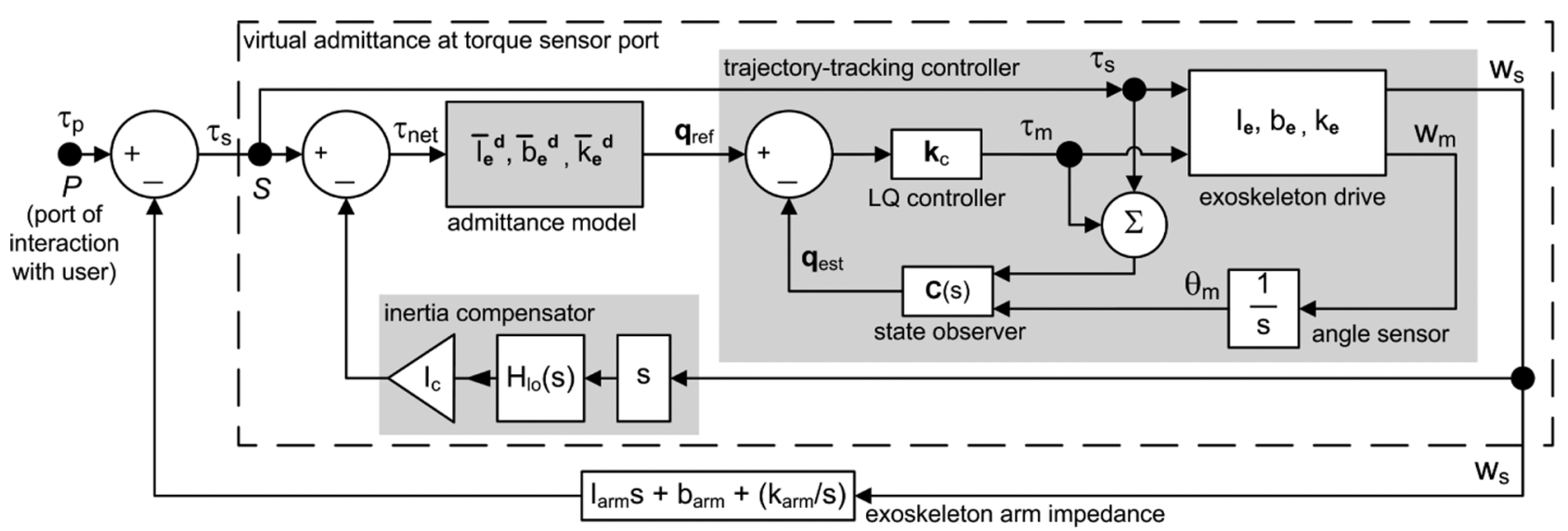

Fig. 2. Detailed model of the exoskeleton controller. A virtual admittance model with impedance parameters $\bar{I}_{e}^{d}, \bar{b}_{e}^{d}$, and $\bar{k}_{e}^{d}$ [see (1)] generates a reference state trajectory $\mathbf{q}_{\text {ref. }}$. The input to the admittance model is the sum of the torque sensor measurement $\tau_{s}$ plus the feedback torque from the inertia compensator. The reference trajectory $\mathbf{q}_{\text {ref }}$ is tracked by a closed-loop controller that uses an LQ regulator. The exoskeleton drive has inertia $I_{e}$, damping $b_{e}$ and stiffness $k_{e}$. The drive's outputs are the angular velocity $w_{m}$ of the servo motor reflected on the output shaft, and the output shaft's own angular velocity $w_{s}$. The servo motor's angle $\theta_{m}$ is measured by a proprietary feedback device that emulates an encoder. A state observer with a Kalman filter is employed to compute a full state estimate for feedback. In the inertia compensator, the angular acceleration feedback signal is low-pass filtered by a fourth-order Butterworth filter $\left(H_{l o}(s)\right)$ with a cutoff frequency of $4 \mathrm{~Hz}$. A negative feedback gain $I_{c}$ emulates a negative inertia term at low frequencies.

uses feedback of the low-pass filtered angular acceleration of the exoskeleton arm. Although this technique does not attain an exact cancellation of the human limb's inertia, it does produce some of its desirable effects, particularly an increase in the pendulum frequency of the leg.

The controller for the physical 1-DOF exoskeleton, implemented in the QNX real-time operating system, is shown in Fig. 2. Its major components are an admittance controller and a feedback loop forming the inertia compensator. The admittance controller consists of an admittance model followed by a trajectory-tracking linear-quadratic (LQ) controller with an error-integral term. The admittance model uses numerical integration to generate the reference state-space trajectory $\mathrm{q}_{\mathrm{ref}}(t)$ that will be tracked by the closed-loop LQ controller. Kinematic feedback consists of the servo motor's angle $\theta_{m}$, measured by the emulated encoder. A state observer with a Kalman filter $\mathbf{C}(s)$ is provided to compute an estimate of the full feedback state [18].

In order to emulate inertia compensation, angular acceleration is measured by the IMU and low-pass filtered by means of a fourth-order Butterworth filter with a transfer function $H_{l o}(s)$. The cutoff frequency of the filter is $4 \mathrm{~Hz}$. Given the location of the torque sensor (port $S$ in Fig. 2), the inertia felt by the user when $I_{c}=0$ is the sum of the physical inertia of the exoskeleton's arm, $I_{\text {arm }}\left(0.185 \mathrm{~kg}-\mathrm{m}^{2}\right)$, plus the baseline virtual inertia generated by the admittance controller, $\bar{I}_{e}^{d}$ (set to $0.035 \mathrm{~kg}-\mathrm{m}^{2}$ in the experiments presented here). So in theory the emulated inertia compensator has to counteract a total inertia $\bar{I}_{e}^{d}+I_{\text {arm }}\left(0.22 \mathrm{~kg}-\mathrm{m}^{2}\right)$ before it can compensate the inertia of the leg itself ${ }^{1}$. The selected cutoff frequency represents a design compromise between frequency content and phase lag. At higher cutoff frequencies, the frequency content introduced by the compliance of the ankle coupling makes it difficult to control

\footnotetext{
${ }^{1} \mathrm{~A}$ consequence of noncollocation is that there is no advantage in changing the location of the torque sensor. A previously published result [18] shows that, if we reduce the amount of physical inertia between the torque sensor and the point of contact with the human limb (for example by placing the torque sensor at the ankle brace), then the virtual inertia $\bar{I}_{e}^{d}$ has to be increased by the same amount in order to maintain stability in the baseline condition.
}

voluntary leg movements. An excessively low cutoff frequency, on the other hand, reduces the fidelity of the inertia compensation effect due to phase lag.

\section{Assistive Action of the Exoskeleton in Terms of Impedance}

An analysis of the exoskeleton's impedance at the interaction port $P$ (Fig. 2) shows that emulated inertia compensation combines two different assistive effects. It increases the natural frequency of the coupled system by means of the inertia compensation effect, and also makes the exoskeleton perform net positive work on the limb on every swing cycle. This net work is due to the negative real part of the exoskeleton's impedance $Z_{e}^{p}(j \omega)$ at the interaction port $P$. Fig. 3 shows parametric plots of $Z_{e}^{p}(j \omega)$ for different swing frequencies $f_{s}$ and different inertia compensation gains $I_{c}$. The effect of the inertia compensation gain $I_{c}$ on the system can be understood by observing the behavior of $Z_{e}^{p}(j \omega)$ at a given swing frequency, for example $f_{s}=0.75 \mathrm{~Hz}$, and different values of $I_{c}$. At $I_{c}=0$ the exoskeleton behaves as a pure inertia, therefore $Z_{e}^{p}(j \omega)$ is a pure imaginary number. As $I_{c}$ becomes more negative, $\operatorname{Im}\left\{Z_{e}^{p}(j \omega)\right\}$ is observed to decrease. This effect is approximately equivalent to a reduction in the inertia of the exoskeleton. At the same time, it can be seen that the real part of $Z_{e}^{p}(j \omega)$ takes on increasingly negative values. Thus $\operatorname{Re}\left\{Z_{e}^{p}(j \omega)\right\}$ can be thought of as a negative damping term that varies with $\omega$. This term causes the exoskeleton to transfer net energy to the human leg, rather than draw energy from it as an ordinary damper would.

Furthermore, it is possible to make the imaginary part of $Z_{e}^{p}(j \omega)$ negative as well if $I_{c}$ is sufficiently large in magnitude. Fig. 3 shows that, for $I_{c}=-1.5 \bar{I}_{e}, \operatorname{Im}\left\{Z_{e}^{p}(j \omega)\right\}$ is negative for a range of swing frequencies $f_{s}$ between 0 and about $1.25 \mathrm{~Hz}$. For example, at $f_{s}=0.75 \mathrm{~Hz}$ we have $\operatorname{Im}\left\{Z_{e}^{p}(j \omega)\right\}=$ $-0.33 \mathrm{~N}-\mathrm{m}-\mathrm{s} / \mathrm{rad}$. Thus making $\operatorname{Im}\left\{Z_{e}^{p}(j \omega)\right\}$ negative is, in a sense, equivalent to making the exoskeleton behave as a negative inertia. The expected outcome is that the exoskeleton in this condition will make the natural frequency of the coupled system 


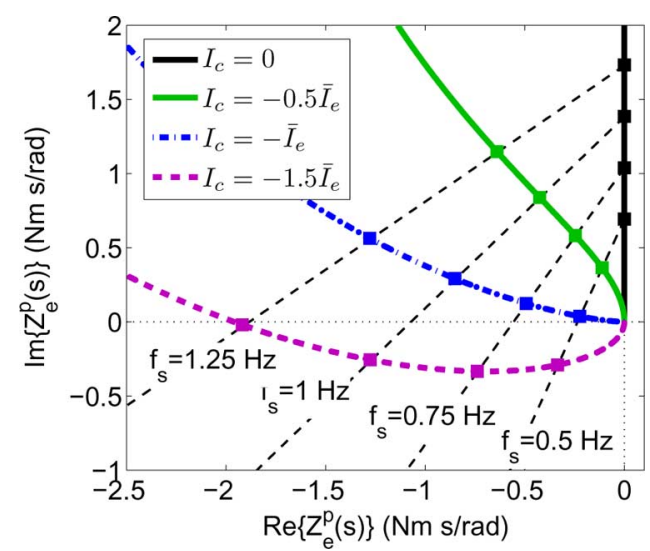

Fig. 3. Parametric plots of the exoskeleton's impedance $Z_{e}^{p}(j \omega)$ at the interaction port, where $\omega=2 \pi f_{s}$ and $f_{s}$ is the leg swing frequency in Hz. Inertia compensation gains $I_{c}$ are expressed as fractions of the exoskeleton's net inertia $\bar{I}_{e}=I_{\mathrm{arm}}+\bar{I}_{e}^{d}$. The thin dotted lines represent curves of equal swing frequency $f_{s}$

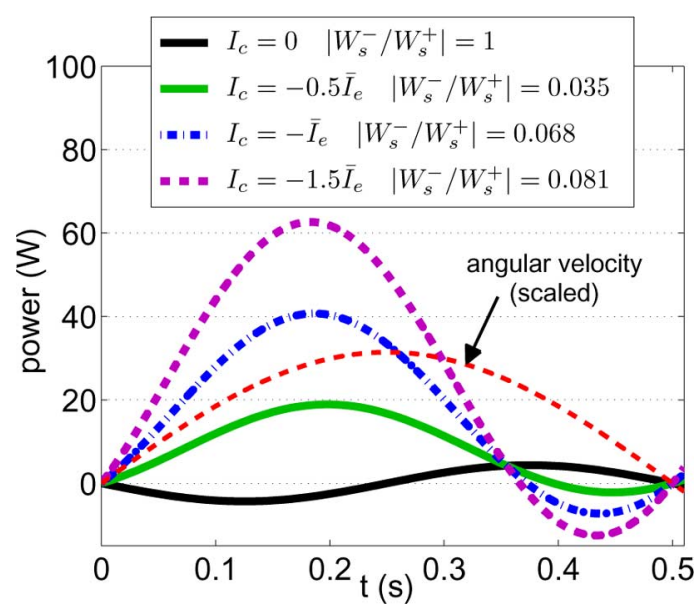

Fig. 4. Instantaneous power transferred at the torque sensor port over half a cycle of leg swing $\left(f_{s}=1 \mathrm{~Hz}\right)$, for different levels of inertia compensation $I_{c}$. Also shown is the ratio of negative to positive work $\left|W_{s}^{-} / W_{s}^{+}\right|$for each value of $I_{c}$. The angular velocity of the coupled limb-exoskeleton is shown for reference.

(human limb and exoskeleton) larger than the natural frequency of the unassisted limb.

An important property of emulated inertia compensation is that the exoskeleton's drive performs mostly positive work during the cycle of leg swing. This fact is illustrated by Fig. 4, which shows plots of the instantaneous power transferred at the torque sensor port (i.e., the output of the exoskeleton's drive) over half a cycle of leg swing at $1 \mathrm{~Hz}$, for different values of $I_{c}$. For $I_{c}=0$, the ratio of negative to positive work at the torque sensor port, $\left|W^{-} / W^{+}\right|$, is equal to 1 , indicating that no net work is performed. This is entirely expected because at $I_{c}=0$ the impedance $Z_{e}^{s}$ of the exoskeleton corresponds to that of a pure inertia of magnitude $\bar{I}_{e}$. However, for negative values of $I_{c}$ the amount of negative work performed becomes quite small, in all cases remaining well below $10 \%$ of the amount of positive work performed. A consequence is that, in a wearable exoskeleton, the actuator can be expected to perform very little "braking" action, thereby avoiding power waste.

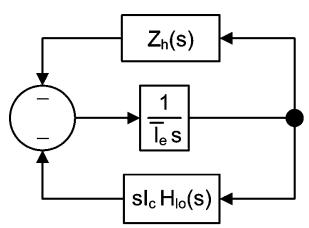

(a)

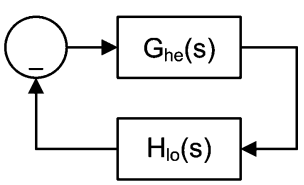

(b)
Fig. 5. Model of the coupled human-exoskeleton for stability robustness analysis. (a) The human limb, represented by impedance $Z_{h}(s)$, is rigidly coupled to the exoskeleton. Assuming ideal trajectory-tracking performance, the exoskeleton is represented as a pure inertia $\bar{I}_{e}$ coupled to the inertia compensator, which has a transfer function $s I_{c} H_{l o}(s)$. (b) Closed-loop equivalent of the system.

\section{Robustly Stable Interaction Between the Exoskeleton and the Human Limb}

The stability of the coupled system formed by the human limb and the exoskeleton has been previously analyzed under the assumption of a rigid coupling between the leg and the exoskeleton [18]. The stability robustness of the coupled humanexoskeleton system in the presence of parameter uncertainties will now be examined. The analysis begins with the sensitivity of the system to variations in the human limb parameters. In order to keep the analysis simple, it is assumed that the controller has ideal trajectory-tracking performance, and that the human limb and the exoskeleton are rigidly coupled. The coupled system formed the exoskeleton and the human limb can thus be represented by the model of Fig. 5(a). In the model shown, $Z_{h}(s)$ is the impedance of the human limb, $\bar{I}_{e}$ is the net inertia of the exoskeleton at the interaction port and $H_{l o}(s)$ is the transfer function of the low-pass Butterworth filter. The system can be represented alternatively by the model in Fig. 5(b), where $G_{h e}(s)$ is given by

$$
G_{h e}(s)=\frac{I_{c}}{I_{h}+\bar{I}_{e}}\left(\frac{s^{2}}{s^{2}+2 \zeta_{h e} \omega_{n, h e} s+\omega_{n, h e}^{2}}\right)
$$

and

$$
\begin{aligned}
\zeta_{h e} & =\zeta_{h} \sqrt{\frac{I_{h}}{I_{h}+\bar{I}_{e}}} \\
\omega_{n, h e} & =\omega_{n, h} \sqrt{\frac{I_{h}}{I_{h}+\bar{I}_{e}}} .
\end{aligned}
$$

$I_{h}$ represents the moment of inertia of the human limb segment, and $\zeta_{h}$ represents the damping ratio of the limb's joint. The terms $\zeta_{h e}$ and $\omega_{n, h e}$ represent, respectively, the damping ratio and the natural frequency that result from adding the exoskeleton's inertia $\bar{I}_{e}$ to the human limb. The transfer function of the Butterworth filter has the property $\left\|H_{l o}(j \omega)\right\|_{\infty} \leq 1$. Therefore, from the small-gain theorem a sufficient condition for coupled stability is

$$
\left\|G_{h e}(j \omega)\right\|_{\infty}=\left\|G_{h e}\left(j \omega_{r, h e}\right)\right\|<1
$$

where $\omega_{r, h e}$ is the resonant frequency of $G_{h e}(j \omega)$, given by

$$
\omega_{r, h e}=\omega_{n, h e} \sqrt{1-2 \zeta_{h e}^{2}} .
$$


By combining (4) and (5), the following robust stability condition can be derived:

$$
-1<\frac{I_{c}}{I_{h}+\bar{I}_{e}}\left(\frac{1-2 \zeta_{h e}^{2}}{2 \zeta_{h e} \sqrt{1-\zeta_{h e}^{2}}}\right)<1 .
$$

Condition (6) gives a range of values of $I_{c}$ for which the stability of the coupled system is guaranteed by the small gain theorem. The lower boundary of this range is

$$
I_{c}>I_{c, \min }
$$

where

$$
I_{c, \min }=-\left(I_{h}+\bar{I}_{e}\right) \frac{2 \zeta_{h e} \sqrt{1-\zeta_{h e}^{2}}}{1-2 \zeta_{h e}^{2}} .
$$

The effect of emulated inertia compensation on the human limb can be understood as multiplying the inertia of the leg segment $I_{h}$ by a factor $\alpha_{i}$ such that $0<\alpha_{i}<1$. In other words, wearing the exoskeleton should produce an effect similar to reducing the human limb's inertia to a fraction $\alpha_{i} I_{h}$. Treating $I_{c}$ as an inertia term, the value of $I_{c}$ that corresponds to a desired value of $\alpha_{i}$ is computed as

$$
I_{c}=\left(\alpha_{i}-1\right) I_{h}-\bar{I}_{e}
$$

Condition (7) in turn determines the following robust stability limit for the virtual inertia reduction represented by $\alpha_{i}$ :

$$
\alpha_{i}>\alpha_{i, \min }
$$

where

$$
\alpha_{i, \min }=\frac{I_{h}+\bar{I}_{e}+I_{c, \min }}{I_{h}} .
$$

Ideally, in order to determine the amount of inertia compensation that can be applied to a specific user, we should know the values of the impedance parameters of the human limb. Fig. 6 shows a plot of $\alpha_{i, \min }$ as a function of the limb segment inertia $I_{h}$ and the net damping ratio of the knee joint, $\zeta_{h e}$. This plot shows that the robust stability of the coupled system is quite sensitive to the damping ratio. In general, the higher the damping of the knee joint, the larger the virtual inertia reduction that can be accomplished without losing stability. Fig. 6 also suggests that subjects with higher limb inertias can accommodate comparatively larger levels of inertia reduction. This observation will be further examined in the experimental part of the study.

\section{E. Experiments With Emulated Inertia Compensation}

1) Race Experiment Definition: An experimental study was conducted to compare between free leg-swing motion, and leg-swing motion using the 1-DOF exoskeleton. A preliminary analysis focusing exclusively on swing frequency has been presented in an earlier paper [18]. Here we present a more comprehensive analysis of the data set from that study, with a focus on the kinematics of leg swing, the net energy transfer produced by the exoskeleton and the possible loss of stability under inertia compensation.
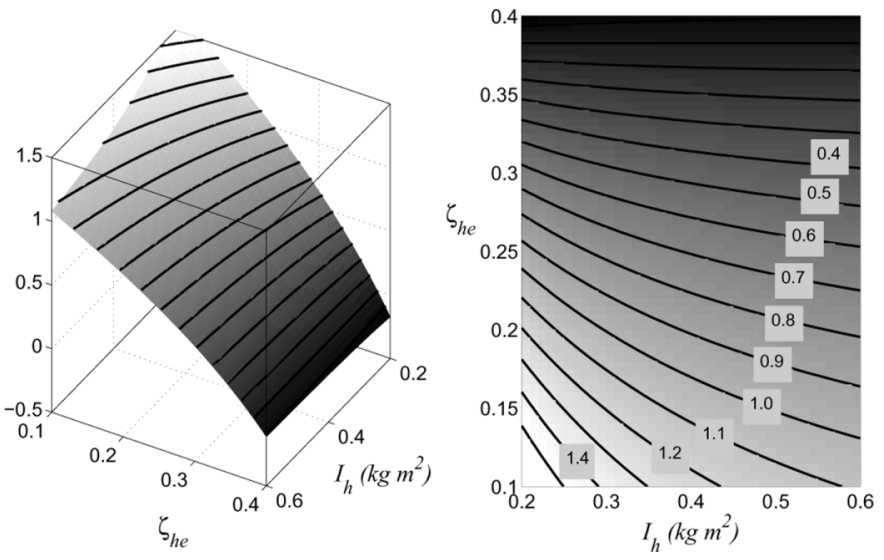

Fig. 6. Sensitivity of the robust stability condition to parameter variations: inertia compensation factor $\alpha_{i, \min }$ as a function of the human limb inertia $I_{h}$ and the net damping ratio of the knee joint, $\zeta_{h e}$.

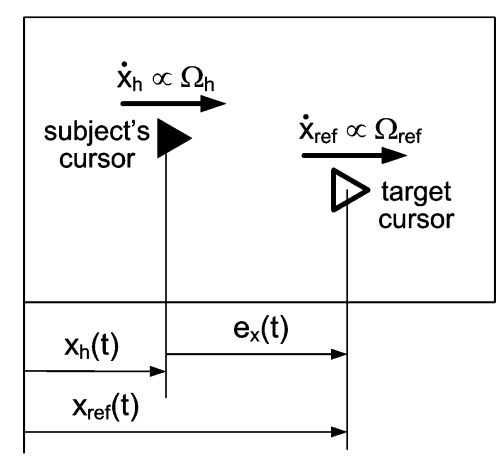

Fig. 7. Graphic user interface for the experimental task. The linear speed $\dot{x}_{h}$ of the subject's cursor is directly proportional to the leg's rms angular velocity $\Omega_{h}$. The linear speed $\dot{x}_{\text {ref }}$ of the subject's cursor is directly proportional to $\Omega_{\text {ref }}$.

The primary objective of the experiments was to determine how the subjects' selected combination of swing frequency and swing amplitude changes when wearing the exoskeleton. Such changes may have their correspondence in changes to step frequency and step length when walking with an autonomous exoskeleton.

The experimental task gives the subject a target value of root mean square (rms) angular velocity, $\Omega_{\text {ref }}$, to be matched or exceeded by swinging the leg. The task has the form of a race against a virtual target; it is presented to the user through a computer graphic interface shown schematically in Fig. 7. The display shows two cursors that traverse the screen from left to right. The subject's cursor moves in response to the swing motion of the subject's leg; its linear speed is directly proportional to the leg's rms angular velocity $\Omega_{h}$. The "target" cursor travels at a constant linear speed proportional to $\Omega_{\text {ref }}$. The leg's rms angular velocity is computed in real time as a running average with a time window of $0.15 \mathrm{~s}$. Subjects are implicitly given freedom to select any combination of frequency and amplitude of leg swing in order to produce $\Omega_{h}$.

The experiment consisted of a series of races between the subject's cursor and the target cursor. The standard duration of a trial was $15 \mathrm{~s}$, except for the catch trials, which had a longer 
duration. The instruction to the subjects was to swing their leg fast enough to make their cursor overtake the target cursor before the end of the trial. For all trials, the velocity of the target cursor, $\Omega_{\text {ref }}$, was set to be $20 \%$ larger than the subject's preferred velocity of unassisted leg swing. In designing the task we considered that, unlike walking, stationary leg swing is an unfamiliar task to most subjects. Therefore, the task has to involve a certain goal in order to produce an identifiable pattern of movement. On the other hand, if the task involves too many constraints, the effect of the exoskeleton might become less evident. Thus, we decided that the goal should be simply to overtake the cursor within the allotted time, but placed no further constraints on how the subject should achieve that goal. In this way, the task places a lower boundary on the subjects' rms angular velocity, but not a higher one.

For the purposes of the present analysis, the last $7.5 \mathrm{~s}$ of the trial are considered to be the "steady-state" phase, i.e., a phase in which variations of $\Omega_{h}$ are at a minimum. However, it is not implied that the rms angular velocity should not display any trend after $7.5 \mathrm{~s}$. For example, subjects might tend to slow down once the goal the target cursor has been overtaken.

2) Subjects: Ten male healthy subjects participated this study (body mass $=72.4 \pm 11.7 \mathrm{~kg}$ (mean \pm s.d.); height $=178 \pm$ $6 \mathrm{~cm}$; age $=22.1 \pm 2.9$ years). None of the subjects had previous experience using the exoskeleton. The experimental protocol was approved by the Institutional Review Board of Northwestern University; all subjects gave their informed consent previous to participating in the experiment.

3) Experimental Conditions: The race task was performed under three different experimental conditions.

- UNCOUPLED: The subject swings the leg unaided. The IMU is temporarily attached to the ankle in order to generate angular velocity and angular acceleration data.

- BASELINE: The subject wears the exoskeleton with zero inertia compensation $\left(I_{c}=0\right)$, thus being subject to the full inertia of the exoskeleton's arm.

- ASSIST: The subject wears the exoskeleton with a specific level of inertia compensation, defined by the gain value $I_{c}$.

The number of trials executed was five in each of the UNCOUPLED and BASELINE conditions, and eleven in the ASSIST condition.

4) Determining the Inertia Compensation Gain: For the ASSIST condition we implemented a procedure to find a borderline destabilizing value for the negative gain $I_{c}$. Each subject underwent a series of calibration trials upon completing the BASELINE trials. The subjects was instructed to swing the leg at a comfortable rhythm while wearing the exoskeleton. The duration of each calibration trial was $15 \mathrm{~s}$. On each trial the subject was exposed to a larger negative value of $I_{c}$. The value of $I_{c}$ employed for the ASSIST trials was the one that produced a first perception of difficulty switching the direction of the leg.

5) Output Variables and Experimental Hypothesis: The output variables for the experiment were the rms angular velocity, the frequency of leg swing and the swing amplitude. For the purposes of statistical analysis, the rms angular velocity was computed using a time window of $3.0 \mathrm{~s}$. Frequency and amplitude were obtained using the Hilbert transform [19]. This method is preferred over computing the fast Fourier transform

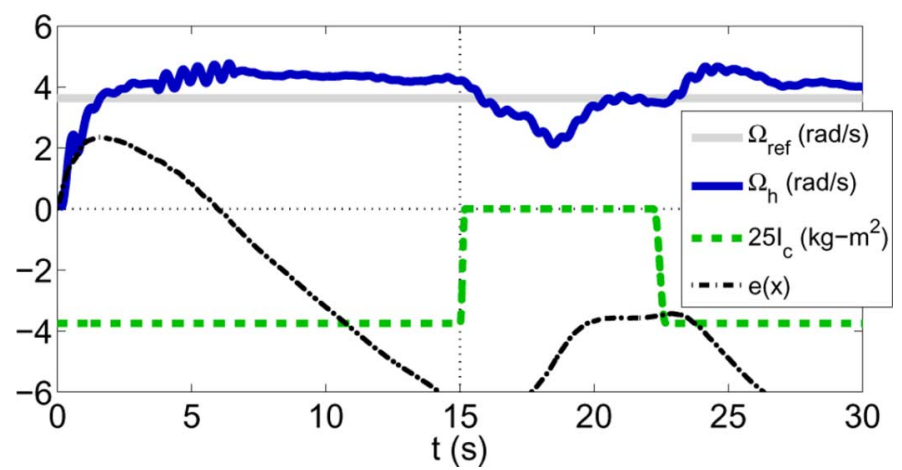

Fig. 8. Time series plots of a typical catch trial for the ASSIST task with inertia compensation. The time plot for the inertia compensation gain $I_{c}$ has been scaled vertically for easier visualization.

due to the nonstationary nature of the signals. The method for computing the swing frequency consisted of decomposing the angular position trajectory of the leg, $\theta(t)$, into a set of components called intrinsic mode functions [20], and applying the Hilbert transform to the lowest-frequency component.

The hypothesis for the race experiments was that in the BASELINE trials the exoskeleton arm's inertia would reduce the steady-state frequency of leg swing in comparison with the UNCOUPLED trials, and that the steady-state frequency would increase again in the ASSIST condition due to the inertia compensation effect. The amplitude of leg swing was expected to increase significantly in the BASELINE condition due to the virtual reduction in the damping ratio of the leg. (Recall that the net effect of the exoskeleton in the BASELINE condition is the addition of pure inertia.) The effect of the ASSIST condition on swing amplitude was harder to predict because emulated inertia compensation combines an increase in natural frequency and negative damping.

6) Catch Trials: In order to evidence the exoskeleton's assistive effect, a number of catch trials were implemented for the ASSIST condition. These corresponded to trials \#4, \#8, and \#11 of the ASSIST sequence. Trials \#4 and \#8 were extended-duration trials; they were intended to show the assistive effect of the exoskeleton during the steady-state phase, particularly the net transfer of energy due to negative real part of the exoskeleton's impedance. Fig. 8 shows exemplary plots of these catch trials for a typical ASSIST sequence. ( $I_{c}$ has been scaled vertically for easier visualization.) At $t=15 \mathrm{~s}$ the inertia compensation gain $I_{c}$ suddenly becomes zero and remains at that value for about $7.5 \mathrm{~s}$, after which the original value of $I_{C}$ is restored. The expected effect, noticeable in Fig. 8, is a reduction in the magnitude of the leg's rms velocity $\Omega_{h}(t)$ during the time interval with $I_{c}=0$, followed by a partial recovery in the magnitude of $\Omega_{h}(t)$ once $I_{c}$ is restored.

Trial \#11 was a trial of normal duration, but with zero inertia compensation applied for the entire duration of the trial. The expected behavior was a reduction in steady-state angular velocity $\Omega_{h}(t)$ with respect to the preceding trial, i.e., trial \#10.

7) Statistics: Repeated-measures ANOVA was performed with experimental condition (UNCOUPLED, BASELINE, or ASSIST) as the factor. The output variables were the steadystate values of rms angular velocity, swing frequency and swing 
amplitude. The steady-state rms angular velocity for a particular subject and experimental condition was computed as the average of consecutive trials ${ }^{2}$. The swing frequency and swing amplitude were treated in the same manner. If the effect of the experimental condition was found to be significant, Tukey honestly significant difference (HSD) tests would then be used to determine specific differences between the means.

\section{RESULTS}

\section{A. Kinematics of Leg Swing: Steady-State Phase}

The net exoskeleton inertia presented to the subjects in the BASELINE condition was $0.22 \mathrm{~kg}-\mathrm{m}^{2}$, which is equal to the sum of the arm inertia $I_{\mathrm{arm}}\left(0.185 \mathrm{~kg}-\mathrm{m}^{2}\right)$ plus the virtual inertia of the drive mechanism, $\bar{I}_{e}^{d}$ (set to $0.035 \mathrm{~kg}-\mathrm{m}^{2}$ for this experiment). This being a first experiment, inertia compensation gains were applied conservatively. The range of values for $I_{c}$ was $-0.125 \pm 0.024 \mathrm{~kg}-\mathrm{m}^{2}$ (mean \pm s.d.). Thus, the net exoskeleton inertia of $0.22 \mathrm{~kg}-\mathrm{m}^{2}$ was not fully compensated for in these experiments.

The experimental conditions were found to have a significant effect on the steady-state rms angular velocity (ANOVA: $p<$ 0.01; HSD: ASSIST > BASELINE, ASSIST > UNCOUPLED) and leg-swing frequency (ANOVA: $p<$ 0.03; HSD: BASELINE < UNCOUPLED, ASSIST > BASELINE). A certain tendency to increase the swing amplitude was observed in the BASELINE and ASSIST conditions, but not at the level of statistical significance. The behaviors of the rms velocity, swing frequency and swing amplitude as a function of time during the race experiment are shown in Fig. 9(a)-(c). The plots provide comparisons between the ASSIST and UNCOUPLED conditions, and between the ASSIST and BASELINE conditions. Subjects in general were slowed down by the BASELINE condition, due most likely to the exoskeleton's arm inertia. The application of inertia compensation in the ASSIST condition enabled the subjects to swing the leg at higher velocities. Although the inertia compensation gains were kept relatively low, the ASSIST condition produced a considerably higher steady-state rms velocity than in the UNCOUPLED case. It can also be noticed in Fig. 9(a) that the rms velocity in the UNCOUPLED case begins to drop after about $10 \mathrm{~s}$ into the trial, which suggests that subjects tended to reduce their effort once their cursor had overtaken the target. By contrast, the rms velocity remained fairly constant in the ASSIST condition, due most likely to the net work performed by the exoskeleton in this condition.

Fig. 9(d)-(f) shows the mean change between experimental conditions (UNCOUPLED, BASELINE, ASSIST) for each of the output variables. Subjects performing the race task in the BASELINE condition showed a nonsignificant variation in their rms angular velocity $(6.20 \pm 2.28 \%)$, relative to the UNCOUPLED case [Fig. 9(d)]. However, the relative contributions of frequency and amplitude to the angular velocity

\footnotetext{
${ }^{2}$ The first trial in each experimental condition was dropped from the computation of the average. Any difficulties that the subject has adapting to a new experimental condition will show especially in the first trial. Therefore, this trial is not considered to be representative of the subject's overall performance for that condition.
}

changed drastically. Swing frequency was considerably reduced $[-12.99 \pm 4.08 \%$, Fig. 9(e)] due to the exoskeleton's arm inertia. This reduction in BASELINE frequency was somewhat compensated by an increase in the mean amplitude of swing [9.52 $\pm 5.30 \%$, Fig. 9(f)].

The ASSIST condition produced a large increase in rms angular velocity relative to the UNCOUPLED case $[26.02 \pm 3.60 \%$, Fig. 9(d)]. The main factor contributing to this effect was a recovery in the swing frequency of the leg. Fig. 9(e) shows that, for the ASSIST condition, the introduction of inertia compensation restored the mean swing frequency to nearly its original value, although actual per-subject means showed considerable variation $(0.88 \pm 6.34 \%)$. Interestingly, this result was achieved with inertia compensation gains $I_{c}$ that in theory were not large enough in magnitude to fully compensate the inertia of the exoskeleton, let alone compensate the inertia of the human limb.

Phase portraits of angular acceleration versus angular speed were employed to study the differences in leg swing under the different experimental conditions. A specific aim was to find indications of loss of stability in the ASSIST condition. Fig. 10 shows examples of phase portraits obtained from one experimental subject. In the UNCOUPLED condition, the shape of the phase portrait indicates that the behavior of the leg segment is quite different from that of a linear pendulum. This is not unexpected because the stiffness of the knee joint is known to vary nonlinearly with flexion angle [21].

The phase portrait for the BASELINE condition differs considerably from the UNCOUPLED case, as it tends towards an elliptical shape. This is most likely due to the exoskeleton behaving as a pure inertia. Additional inertia may have an effect similar to that of a flywheel, smoothing the trajectory of the leg and bringing it closer to a linear behavior. Interestingly, this qualitative behavior was basically repeated in the ASSIST condition, in spite of the destabilizing effect of the inertia compensation. Thus the phase portraits did not provide a clear way to distinguish between the BASELINE and ASSIST conditions.

Angular jerk (i.e., the time derivative of angular acceleration) was found to provide a sharper distinction between the BASELINE and ASSIST. Angular jerk was computed offline by taking the discrete derivative of angular acceleration. The computed derivative was smoothed by low-pass filtering forward and backward in time with a cutoff frequency of $20 \mathrm{~Hz}$. Fig. 11 shows a comparison between the rms values of angular jerk for the different experimental conditions. While no significant difference was found between the BASELINE and UNCOUPLED conditions, the difference between the ASSIST and UNCOUPLED conditions was quite large (ANOVA: $p<0.01$ ).

\section{B. Catch Trials}

Because the exoskeleton transfers net energy to the leg on every cycle of leg swing, it was expected that the sudden removal of this energy supply (by making $I_{c}=0$ ) during the catch trials would cause the leg to slow down. Experimental data showed that the removal of inertia compensation had a highly significant effect on the rms velocity of swing $(p<0.01$; HSD: $\left.\bar{\Omega}_{h}\right|_{I_{c}=0}<\bar{\Omega}_{h, s s}$ for trials \#4 and \#8; no significant difference when comparing trials \#11 and \#10). The mean ratios 
(a)
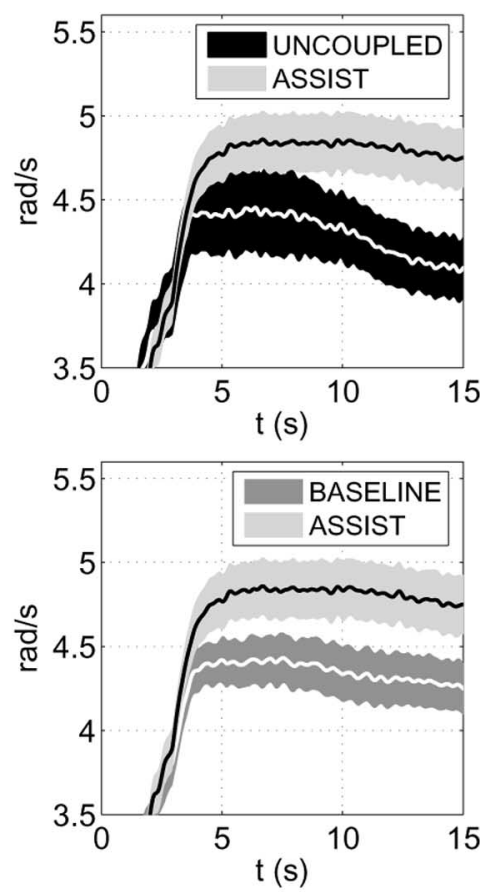

(d)

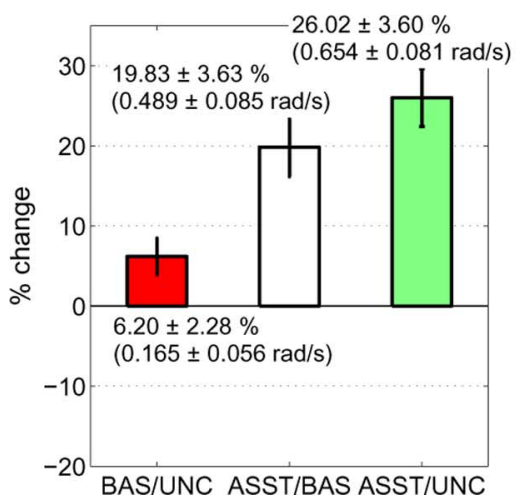

(b)
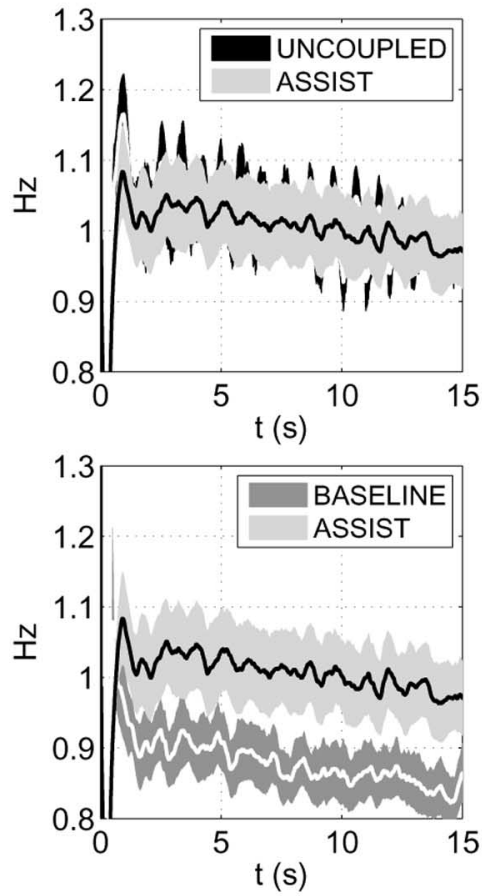

(e)

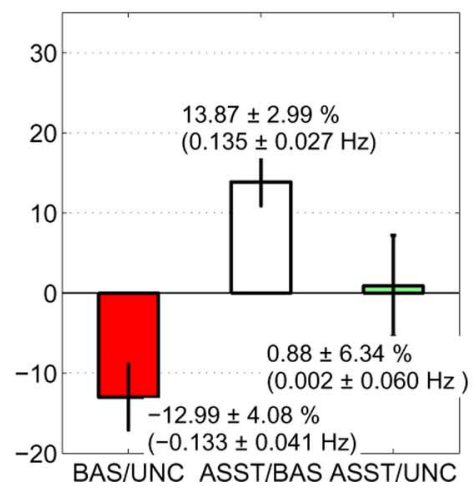

(c)
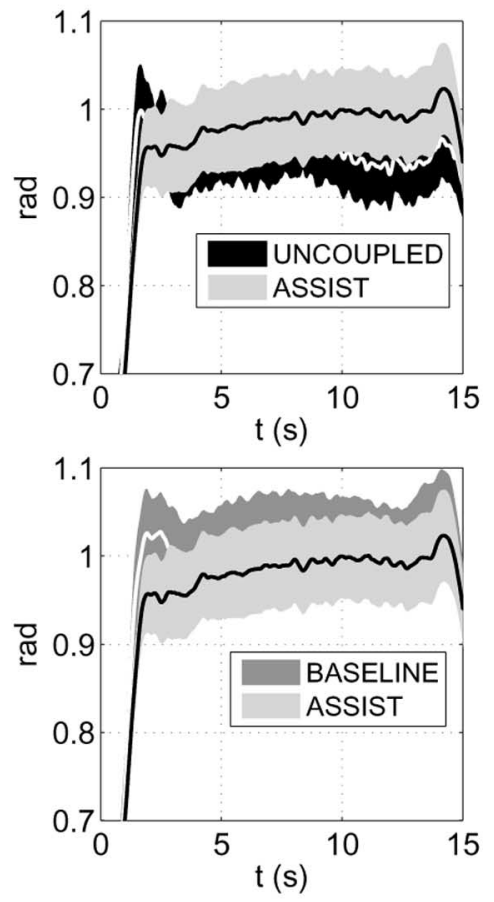

(f)

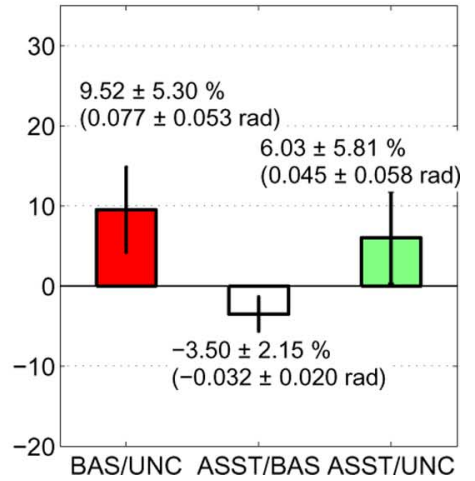

Fig. 9. Kinematics of leg swing for each experimental condition. The first two rows show the comparisons among the time trajectories of the output variables of the race task: (a) rms angular velocity, (b) swing frequency, (c) swing amplitude. Line plots represent the mean values across all subjects and all trials within a particular experimental condition. Shaded regions represent the standard error of the mean. In the bottom row, bars show the percentage change between experimental conditions (UNCOUPLED, BASELINE, ASSIST) for (d) rms angular velocity, (e) swing frequency, and (f) swing amplitude. Error bars are 土s.e.m. Values in parenthesis are the differences (mean \pm s.e.m.) between experimental conditions.

of rms velocity (mean \pm s.e.m.) for the catch trials under the ASSIST condition were as follows.

- Trial \#4: $0.81 \pm 0.02$.

- Trial \#8: $0.84 \pm 0.02$.

- Trial \#11: $0.94 \pm 0.03$.

Thus for trials \#4 and \#8, the sudden removal of inertia compensation (i.e., making $I_{c}=0$ at $t=15 \mathrm{~s}$ ) produced a considerable drop in rms angular velocity. For trial \#11 the difference in rms velocity with respect to trial \#10 was not found to be significant, which suggests that subjects compensated for the lack of exoskeleton assistance during that last trial.

\section{DISCUSSION}

The control method presented here is based on shaping the impedance on the exoskeleton at the port of interaction with the user. The idea of shaping the dynamics of the exoskeleton has been applied before in the "subject comfort" control of the HAL exoskeleton [22], and in the generalized elasticities method proposed by Vallery [23]. However, in those methods the exoskeleton's impedance is passive, therefore no net transfer of energy to the user's limbs will occur unless a layer of active control is added. By contrast, our emulated inertia compensation method makes the exoskeleton exhibit active behavior in order to perform net work on the human limbs. Our method is similar to the control of the BLEEX exoskeleton [5] in that our device employs positive feedback to increase sensitivity to the forces exerted by the user. However, in the case of BLEEX the goal of the controller is merely to scale down the impedance of the exoskeleton's mechanism by certain factor. Therefore the exoskeleton remains passive, although close to instability. (The assistive effect of BLEEX consists of gravitational support of an external load.) 

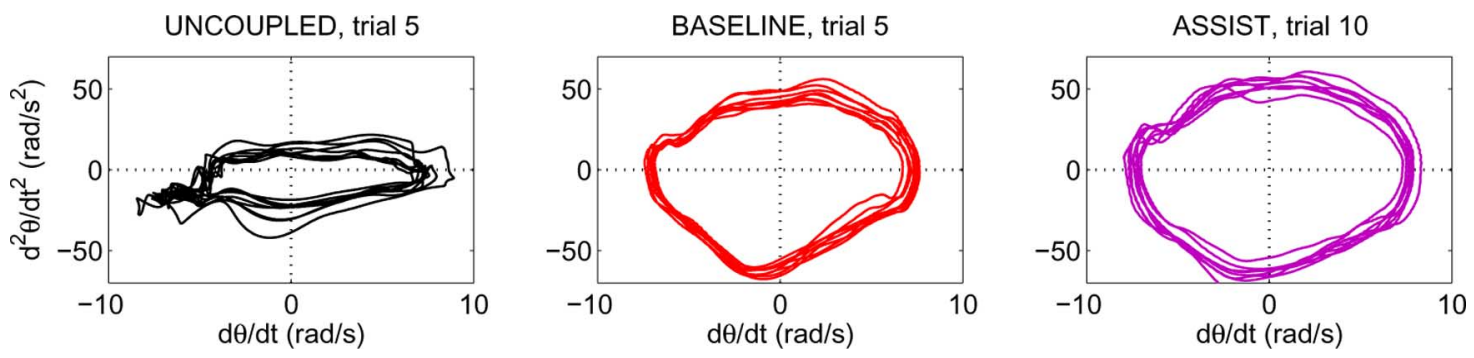

Fig. 10. Examples of phase portraits of angular acceleration versus angular velocity. Each plot represents a trial from the same subject under a different experimental condition (UNCOUPLED, BASELINE, and ASSIST). Each phase portrait shows the last eight cycles of leg swing from the trial, ending at $t=15 \mathrm{~s}$.

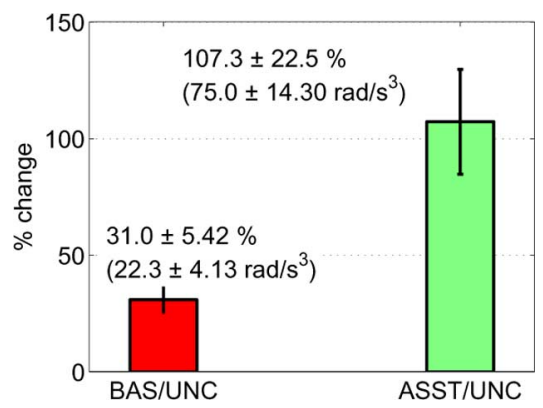

Fig. 11. Angular jerk: percentage change between experimental conditions (UNCOUPLED, BASELINE, ASSIST). All percentages are computed with respect to the variable's mean value in the UNCOUPLED condition. Error bars are \pm s.e.m. Values in parenthesis are the differences (mean \pm s.e.m.) between experimental conditions.

The key results from our experimental study can be summarized as follows:

- In the BASELINE condition, the frequency of leg swing and the mean angular velocity were consistently reduced due to the exoskeleton's inertia.

- Inertia compensation in the ASSIST condition enabled subjects to recover their normal frequency and increase their selected angular velocity.

- On average, the "recovered" frequency was higher than might have been expected from the inertia compensation gains employed.

- Subjects tended to swing at higher amplitudes when wearing the exoskeleton in either condition.

The experimental results support our hypothesis that the frequency of leg swing should decrease in the BASELINE condition due to the inertia added by the exoskeleton. In the ASSIST condition, subjects basically recovered their normal frequency of leg swing due to the inertia compensation effect. A less expected result was that the restoration of the swing frequency was achieved with inertia compensation gains that on average were $43 \%$ smaller than the theoretical value needed to fully compensate the inertia of the exoskeleton. This larger-than-expected increase in frequency may be explained by a higher level of co-contraction of the knee flexor and extensor groups. Increased co-contraction tends to increase the stiffness of the leg joint, thereby making an additional contribution the natural frequency of the limb segment. Future experiments should verify whether higher co-contraction actually takes place, for example through EMG measurements.
A tendency to swing the leg at higher amplitudes was also observed in the BASELINE and ASSIST conditions, although not at the level of significance. In preparatory tests we have observed that subjects tend to swing their legs at a higher amplitude whenever they use the exoskeleton in BASELINE condition. This effect can be easily explained by the fact that the exoskeleton behaves as a pure inertia. Adding extra inertia to the limb segment (with the other impedance parameters remaining the same) produces an apparent reduction in the damping ratio of the knee joint, and consequently a tendency to swing wider. In the ASSIST condition, the effect of the exoskeleton's impedance on the amplitude of swing is more complex. While the virtual reduction in inertia would by itself cause an apparent increase in the damping ratio, the negative damping introduced by the real part of the exoskeleton's impedance tends to produce the opposite effect.

In general, rms angular velocity in the ASSIST condition increased beyond the level that might be expected from the sole recovery of swing frequency. Our interpretation of this fact is that, in addition to inertia compensation, net work due to negative damping is also an important contributor to the speed of leg swing. That effect was evidenced by catch trials \#4 and \#8. The sudden removal of inertia compensation (i.e., making $I_{c}=0$ ) caused the rms angular velocity to drop, which indicates that subjects relied on the exoskeleton's torque contribution to achieve their desired velocity of leg swing.

The method employed to set the inertia compensation gain $I_{c}$ in the ASSIST condition had the drawback of being highly subjective, as it was based on the participant's perception of reduced stability. It is desirable to have an objective method for determining the values of $I_{c}$ that can be safely applied to the user. One alternative approach, based on the results of Section II-D, would be to identify the impedance of the subject's leg segment, with the idea of indexing the inertia compensation gain to the identified human inertia. Computed angular jerk may provide another method for determining $I_{c}$. Statistical analysis showed that angular jerk is quite sensitive to the use inertia compensation, whereas at zero inertia compensation it is not significantly different from the uncoupled case. Therefore, it may be possible to set the maximum inertia compensation gain by defining a maximum acceptable level of rms angular jerk, and leading the subject's response toward that maximum by gradually making $I_{c}$ more negative.

In conclusion, experimental results suggest that emulated inertia compensation can counteract the adverse effects of the exoskeleton's inertia on leg swing frequency and, by extension, 
increase the mean angular velocity of leg swing. However, the effect of inertia compensation on muscle co-contraction levels has yet to be determined. A next step in research will be to test the emulated inertia compensation method on a wearable exoskeleton designed to assist walking.

\section{REFERENCES}

[1] S. Jezernik, G. Colombo, and M. Morari, "Automatic gait-pattern adaptation algorithms for rehabilitation with a 4-DOF robotic orthosis," IEEE Trans. Robot. Automat., vol. 20, no. 3, pp. 574-582, Jun. 2004.

[2] S. Banala, S. Kim, S. Agrawal, and J. Scholz, "Robot assisted gait training with active leg exoskeleton (ALEX)," IEEE Trans. Neural Syst. Rehabil. Eng., vol. 17, no. 1, pp. 2-8, Feb. 2009.

[3] J. Veneman, R. Kruidhof, E. Hekman, R. Ekkelenkamp, E. Van Asseldonk, and H. van der Kooij, "Design and evaluation of the LOPES exoskeleton robot for interactive gait rehabilitation," IEEE Trans. Neural Syst. Rehabil. Eng., vol. 15, no. 3, pp. 379-386, Sep. 2007.

[4] H. Kawamoto and Y. Sankai, "Power assist method based on phase sequence and muscle force condition for HAL," Adv. Robot., vol. 19, no. 7, pp. 717-734, 2005.

[5] H. Kazerooni, J. Racine, R. Huang, and L. Steger, "On the control of the berkeley lower extremity exoskeleton (BLEEX)," in Proc. IEEE Int. Conf. Robot. Automat. (ICRA 2005), Barcelona, Spain, Apr. 18-22, 2005, pp. 4353-4360.

[6] D. Ferris, G. Sawicki, and M. Daley, "A physiologist's perspective on robotic exoskeletons for human locomotion," Int. J. Humanoid Robot., vol. 4, pp. 507-528, 2007.

[7] A. Dollar and H. Herr, "Lower extremity exoskeletons and active orthoses: Challenges and state of the art," IEEE Trans. Robot., vol. 24, no. 1, pp. 144-158, Feb. 2008.

[8] D. Ferris, G. Sawicki, and A. Domingo, "Powered lower limb orthoses for gait rehabilitation," Top Spinal Cord Inj. Rehabil., vol. 11, no. 2, pp. 34-49, 2005.

[9] C. Walsh, D. Paluska, K. Pasch, W. Grand, A. Valiente, and H. Herr, "Development of a lightweight, underactuated exoskeleton for load-carrying augmentation," in Proc. IEEE Int. Conf. Robot. Automat. (ICRA 2006), Orlando, FL, May 15-19, 2006, pp. 3485-3491.

[10] G. Sawicki and D. Ferris, "Mechanics and energetics of level walking with powered ankle exoskeletons," J. Exp. Biol., vol. 211, pp. 1402-1413, 2008.

[11] J. Norris, K. P. Granata, M. R. Mitros, E. M. Byrne, and A. P. Marsh, "Effect of augmented plantarflexion power on preferred walking speed and economy in young and older adults," Gait Posture, vol. 25, pp. 620-627, 2007.

[12] R. C. Browning, J. R. Modica, R. Kram, and A. Goswami, "The effects of adding mass to the legs on the energetics and biomechanics of walking," Med. Sci. Sports Exercise, vol. 39, no. 3, pp. 515-525, 2007.

[13] T. D. Royer and P. E. Martin, "Manipulations of leg mass and moment of inertia: Effects on energy cost of walking," Med. Sci. Sports Exercise, vol. 37, no. 4, pp. 649-656, 2005.

[14] G. Aguirre-Ollinger, J. Colgate, M. Peshkin, and A. Goswami, "Activeimpedance control of a lower-limb assistive exoskeleton," in IEEE 10th Int. Conf. Rehabil. Robot. (ICORR 2007), Noordwijk, The Netherlands, Jun. 13-15, 2007, pp. 188-195.

[15] G. Aguirre-Ollinger, Active Impedance Control of a Lower-Limb Assistive Exoskeleton. Evanston, IL: Northwestern Univ., 2009.

[16] G. Aguirre-Ollinger, J. Colgate, M. Peshkin, and A. Goswami, "A 1-DOF assistive exoskeleton with virtual negative damping: Effects on the kinematic response of the lower limbs," in IEEE/RSJ Int. Conf. Intell. Robots Syst. (IROS 2007), San Diego, CA, Oct.-Nov. 29-2, 2007, pp. 1938-1944.

[17] W. Newman, "Stability and performance limits of interaction controllers," J. Dynamic Syst., Measure. Control, vol. 114, no. 4, pp. 563-570, 1992.

[18] G. Aguirre-Ollinger, J. Colgate, M. Peshkin, and A. Goswami, "Design of an active one-degree-of-freedom lower-limb exoskeleton with inertia compensation," Int. J. Robot. Res., vol. 30, no. 4, 2011.
[19] G. Aguirre-Ollinger, J. Colgate, M. Peshkin, and A. Goswami, "A one-degree-of-freedom assistive exoskeleton with inertia compensation: Effects on the agility of leg swing motion," Proc. Inst. Mechan. Eng., Part H, J. Eng. Med., vol. 225, no. 3, 2011.

[20] N. Huang, Z. Shen, S. Long, M. Wu, H. Shih, Q. Zheng, N. Yen, C. Tung, and H. Liu, "The empirical mode decomposition and the Hilbert spectrum for nonlinear and non-stationary time series analysis," Proc. R. Soc. Lond. A, vol. 454, pp. 903-995, 1998.

[21] L. Zhang, G. Nuber, J. Butler, M. Bowen, and W. Rymer, "In vivo human knee joint dynamic properties as functions of muscle contraction and joint position," J. Biomechan., vol. 31, pp. 71-76, 1998.

[22] S. Lee and Y. Sankai, "Power assist control for leg with HAL-3 based on virtual torque and impedance adjustment," in IEEE Int. Conf. Syst., Man Cybernet., 2002, vol. 4, pp. 6-6.

[23] H. Vallery, A. Duschau-Wicke, and R. Riener, "Generalized elasticities improve patient-cooperative control of rehabilitation robots," in IEEE Int. Conf. Rehabil. Robot. (ICORR 2009), Kyoto, Japan, Jun. 23-26, 2009, pp. 535-541.

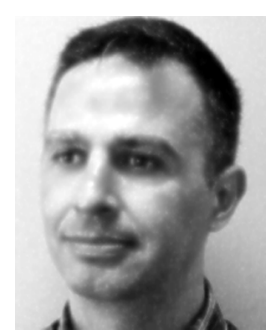

Gabriel Aguirre-Ollinger (M'10) received the $\mathrm{Ph} . \mathrm{D}$. degree in mechanical engineering from Northwestern University, Evanston, IL, in 2009.

$\mathrm{He}$ is a Lecturer with a research-oriented appointment at the School of Electrical, Mechanical and Mechatronic Systems, University of Technology, Sydney, Australia. His research focuses on physical human-robot interaction and rehabilitation robotics.

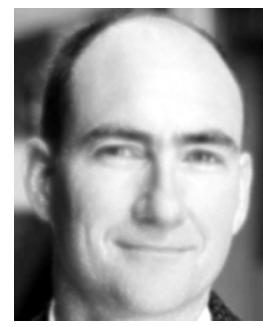

J. Edward Colgate (SM'09) is the Breed University Professor at Northwestern University, Evanston, IL. He has worked extensively in haptic interface, and he is the co-inventor of "cobots," a class of collaborative robots. He is the founder of two start-up companies.

Dr. Colgate is the founding Editor-in-Chief of the IEEE TRANSACTIONS ON HAPTICS.

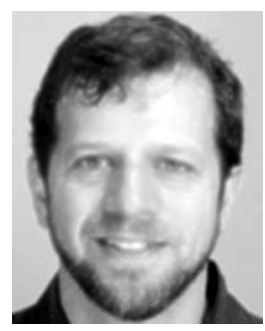

Michael A. Peshkin (SM'09) is a Professor of mechanical engineering at Northwestern University, Evanston, IL. His research is in robotics, human-machine interaction, and rehabilitation robotics. He has cofounded three start-up companies: Mako Surgical, Cobotics, and Kinea Design. He holds 15 patents and is a coinventor (with J. E. Colgate) of cobots.

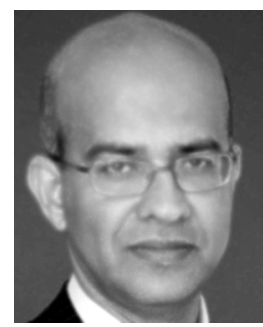

Ambarish Goswami (SM'08) received the Ph.D. degree from Northwestern University, Evanston, IL.

$\mathrm{He}$ has been with Honda Research Institute, Mountain View, CA, for the past nine years, where he is currently a Principal Scientist. His field is dynamics and control, and his main research is in balance maintenance and fall for the Honda humanoid robot ASIMO. 